\title{
Viscosidades de la mezcla n,n-Dimetilformamida + 1-Butanol a Diferentes Temperaturas
}

\author{
Manuel S. Páez ${ }^{(1)^{*}}$, Plinio D. Cantero(1) y Juan F. Peña ${ }^{(2)}$ \\ (1) Universidad de Córdoba, Departamento de Química, Facultad de Ciencias Básicas, \\ Carrera 6 No. 76-103, Km 3, vía Cereté. Córdoba, Colombia. e-mail: (mspaezm@unal.edu.co.) \\ (2) Universidad de Córdoba, Departamento de Física, Facultad de Ciencias Básicas, \\ Carrera 6 No. 76-103, Km 3, vía Cereté. Córdoba, Colombia. \\ * Autor a quien debe dirigirse la correspondencia.
}

Recibido Oct. 19, 2011; Aceptado Dic. 13, 2011; Versión final recibida Dic. 30, 2011

\begin{abstract}
Resumen
Se determinaron los tiempos de flujo de la mezcla binaria usando un viscosímetro Anton Paar modelo AMVn. La mezcla estudiada fue $n$,n-dimetilformamida+1-butanol sobre todo el intervalo de fracciones molares a las siete temperaturas, desde 283.15 a $313.15 \mathrm{~K}$. A partir de los datos obtenidos se calcularon las viscosidades absolutas, las desviaciones de viscosidad, los coeficientes de la ecuación de Redlich-Kister, los parámetros de activación del flujo viscoso y las propiedades termodinámicas de exceso. Los resultados se interpretaron en términos de las interacciones presentes en la solución. El sistema mostró desviaciones negativas de viscosidad, lo cual podría indicar disrupción de los enlaces de hidrógeno presentes en el alcohol y presencia de débiles interacciones físicas entre las moléculas distintas en la mezcla. Esto está de acuerdo con los valores obtenidos para los parámetros de activación de exceso del flujo viscoso.
\end{abstract}

Palabras clave: viscosidad, parámetros de activación, flujo viscoso, propiedades de exceso

\section{Viscosities of the Mixture n,n-Dimethylformamide + 1-Butanol at Different Temperatures}

\begin{abstract}
Flowing times of a binary mixture were determined using an AMVn Anton Paar viscometer. The mixture studied was n, n-Dimetilformamida + 1-butanol over the entire range of mole fractions at seven temperatures, from 283.15 to $313.15 \mathrm{~K}$. From the data obtained, the absolute viscosity, viscosity deviations, coefficients of the Redlich-Kister equation, activation parameters of viscous flow and excess thermodynamic properties were calculated. The results were interpreted in terms of the interactions present in the solution. The system showed negative deviations for the viscosity, which could mean disruption of hydrogen bonds present in the alcohol and the presence of weak physical interactions between unlike molecules in the mixture. This is in agreement with the values obtained for the excess activation parameters of viscous flow.
\end{abstract}

Keywords: viscosity, activation parameters, viscous flow, excess properties 


\section{INTRODUCCIÓN}

La viscosidad es una propiedad de transporte que es importante tanto desde el punto vista teórico como práctico.Es por ello que estudios sistemáticos de las propiedades físicas de mezclas líquidas multicomponentes sean requeridos para permitir un mejor conocimiento de la teoría del estado líquido. Adicionalmente, los datos experimentales de viscosidad son esenciales en muchos cálculos de ingeniería química relacionados con el flujo de fluidos, transferencia de calor y masa.

De igual forma esta propiedad termofísica juega un papel relevante en procesos de tratamientos de aguas residuales, eliminación de residuos químicos, control de contaminantes, corrosión, recuperación de petróleo, entre otros. Un número significativo de estudios se han realizado para medir las viscosidades de una gran variedad de mezclas líquidas; no obstante existe la necesidad de continuar ampliando el banco de datos termodinámicos en este tipo de sistemas en un amplio intervalo de composición y temperatura (Yang et al.,2008; Páez et al.,2011; Bhuiyan et al.,2008).

La N,N-dimetiformamida (DMF) y el 1-Butanol son compuestos de gran importancia a nivel científico e ingenieril. La DMF se caracteriza por poseer un momento dipolar grande $(\mu=3,24$ Debye) y una constante del dieléctrica alta $(\varepsilon=36,71)$ que le confieren la capacidad de ser un excelente solvente para la síntesis de polímeros y productos farmacéuticos(Bhuiyan et al.,2008). Además puede utilizarse como disolvente para sales o compuestos con un peso molecular elevado, debido a la habilidad que posee para formar complejos. Por otra parte, el 1-Butanol es un líquido polar fuertemente asociado mediante puentes de hidrógeno y es de gran interés a nivel industrial, ya que es usado como solvente en una gran variedad de procesos como lo constituyen la producción de tintas de impresión, destilaciones azeotrópicas, formulación de detergentes, extracción de grasas y aceites entre otros (Perry et al., 1992).

En este orden de ideas, el estudio de una propiedad termofísica como la viscosidad en mezclas de amida + alcohol es de gran interés debido a que este tipo de sistemas constituye un modelo apropiado para el estudio de las interacciones en péptidos y proteína-solvente (Kumar et al., 2008). Es por ello, que en este trabajo se indaga acerca de las interacciones soluto-solvente en la mezcla binaria DMF+1-Butanol, a partir de propiedades viscosimétricas a las temperaturas de estudio.

\section{MATERIALES Y MÉTODOS}

Los reactivos usados en este trabajo son los siguientes: 1-Butanol (99.5 \% de pureza) N,NDimetilformamida (99.9\% de pureza), los cuales fueron obtenidos de la casa comercial Merck. En ambos casos se determinó el contenido de agua por el método Karl Fisher.

La preparación de las soluciones fue realizada mediante el método de las pesadas mezclando masas conocidas de los líquidos puros antes mencionados, y fueron preparadas en botellas herméticamente selladas para minimizar pérdidas por evaporación. Todas las medidas de masa fueron hechas en una balanza OHAUS con una sensibilidad de $\pm 10^{-4} \mathrm{~g}$.

Las medidas de la viscosidad absoluta fueron hechas con un viscosímetro Anton Paar modelo AMVn. El principio de medición de este equipo se basa en la ley de Stoke. Así, la viscosidad de líquidos puros y su composición es determinada observando el tiempo requerido para que una esfera sólida (acero), recorra una distancia fija entre los dos sensores inductivos en un tubo cilíndrico inclinado que contiene la muestra.

El viscosímetro arroja los tiempos de flujo y calcula automáticamente la viscosidad absoluta previa incorporación de la densidad de la muestra las cuales fueron obtenidas previamente utilizando un densímetro de tubo vibratorio Anton Paar modelo DMA 5000. Así es posible tomar medidas en un amplio intervalo de viscosidad con una exactitud de $\pm 0.01 \mathrm{~K}$, garantizada mediante un termostato tipo Peltier. 
El método de trabajo tiene las siguientes especificaciones: diámetro del tubo capilar $(1.6 \mathrm{~mm})$, densidad de la esfera de acero $\left(7.85 \mathrm{~g}_{\mathrm{cm}} \mathrm{cm}^{-3}\right)$, ángulo de inclinación del tubo capilar con respecto a la horizontal $\left(50^{\circ}\right)$. Estos detalles son de fundamental importancia para un desarrollo adecuado de las medidas. La calibración del viscosímetro fue realizada utilizando líquidos puros de referencia (metanol, etanol, 1-Propanol, 1-Butanol y 1-Pentanol). Las viscosidades de estos fluidos fueron obtenidas a partir de la literatura (Riddick et al., 2008). El método empleado permite obtener medidas de viscosidad con una incertidumbre experimental de $\pm 0.002 \mathrm{mPa} \cdot \mathrm{s}$.

\section{RESULTADOS Y DISCUSIÓN}

Las desviaciones de viscosidad fueron calculadas usando la ecuación (1)

$$
\Delta \eta=\eta-\left(x_{1} \eta_{1}+x_{2} \eta_{2}\right)
$$

Donde $\eta, \eta_{1}, \eta_{2}, x_{1}$ y $x_{2}$ son respectivamente la viscosidad absoluta de la mezcla, la viscosidad de los componentes puros y las fracciones molares.

Los resultados experimentales obtenidos se muestran en la tabla 1 y los valores de la desviación de viscosidad $\Delta \eta$ en función de la fracción molar del 1-Butanol se presentan en la figura 1.

Tabla 1: Viscosidades de mezclas de DMF $\left(\mathrm{x}_{1}\right)$ con 1-Butanol $\left(\mathrm{x}_{2}\right)$ a temperaturas desde $283.15 \mathrm{~K}$ hasta $313.15 \mathrm{~K}$

\begin{tabular}{cccccccc}
\hline $\mathrm{T} / \mathrm{K}$ & 283.15 & 288.15 & 293.15 & 298.15 & 303.15 & 308.15 & 313.15 \\
\hline $\mathrm{x}_{2}$ & \multicolumn{7}{c}{$\eta / \mathrm{mPa} . \mathrm{s} \pm 0.002$} \\
\hline 0.0000 & 0.989 & 0.922 & 0.863 & 0.808 & 0.760 & 0.716 & 0.675 \\
0.0100 & 1.006 & 1.007 & 1.007 & 1.007 & 1.007 & 1.008 & 1.008 \\
0.0305 & 1.023 & 1.023 & 1.023 & 1.022 & 1.022 & 1.022 & 1.021 \\
0.0501 & 1.037 & 1.036 & 1.034 & 1.032 & 1.031 & 1.031 & 1.031 \\
0.0601 & 1.045 & 1.043 & 1.041 & 1.039 & 1.038 & 1.036 & 1.036 \\
0.0856 & 1.059 & 1.056 & 1.053 & 1.049 & 1.046 & 1.044 & 1.042 \\
0.1000 & 1.070 & 1.069 & 1.062 & 1.058 & 1.055 & 1.053 & 1.050 \\
0.1498 & 1.119 & 1.114 & 1.106 & 1.097 & 1.090 & 1.088 & 1.082 \\
0.2002 & 1.167 & 1.160 & 1.149 & 1.138 & 1.129 & 1.119 & 1.111 \\
0.2999 & 1.269 & 1.256 & 1.238 & 1.220 & 1.210 & 1.191 & 1.177 \\
0.4000 & 1.389 & 1.369 & 1.341 & 1.315 & 1.297 & 1.275 & 1.257 \\
0.4972 & 1.548 & 1.516 & 1.477 & 1.442 & 1.413 & 1.383 & 1.354 \\
0.5931 & 1.734 & 1.692 & 1.640 & 1.588 & 1.547 & 1.507 & 1.469 \\
0.6988 & 1.986 & 1.926 & 1.857 & 1.786 & 1.729 & 1.674 & 1.620 \\
0.7990 & 2.298 & 2.217 & 2.122 & 2.037 & 1.954 & 1.875 & 1.804 \\
0.8990 & 2.891 & 2.747 & 2.600 & 2.453 & 2.334 & 2.215 & 2.109 \\
1.0000 & 3.940 & 3.675 & 3.412 & 3.171 & 2.972 & 2.772 & 2.593 \\
\hline
\end{tabular}

La tabla 1, muestra que la adición de 1-Butanol al compuesto químico DMF logra incrementar ligeramente la viscosidad de éste hasta que la mezcla alcanza el valor de la viscosidad del 1Butanol puro. Ahora bien, dado que el 1-butanol es poco disociable, este sólo se disocia en la región rica en el alcohol a un ritmo creciente, y como resultado la viscosidad aumenta rápidamente (Mohammad et al.,1999).

El análisis de la figura 1, permite inferir que las desviaciones de viscosidad de la mezcla binaria (DMF)(1)+1-butanol(2) son negativas a todas las temperaturas de estudio y en todo el intervalo de concentración. Adicionalmente se observa que las curvas de desviación de viscosidad muestran un mínimo alrededor de la fracción molar $x_{2}=0.799$. Las desviaciones de viscosidad están relacionadas con las interacciones moleculares, por ejemplo enlaces de hidrógeno, las 
interacciones de transferencia de carga y las interacciones físicas, por ejemplo las fuerzas de dispersión o interacciones débiles dipolo-dipolo. En consecuencia, las desviaciones negativas de la viscosidad del sistema (DMF)(1)+1-Butanol(2) se pueden atribuir a la disrupción de los enlaces de hidrógeno del alcohol y a débiles interacciones físicas entre las especies de la mezcla, (Zivkovi'c et al., 2010, Páez et al.,2009).Resultados similares se han encontrado para mezclas DMF + etanol y DMF + N-metilformamida (Ali et al., 1996; Mohammad et al., 1999).

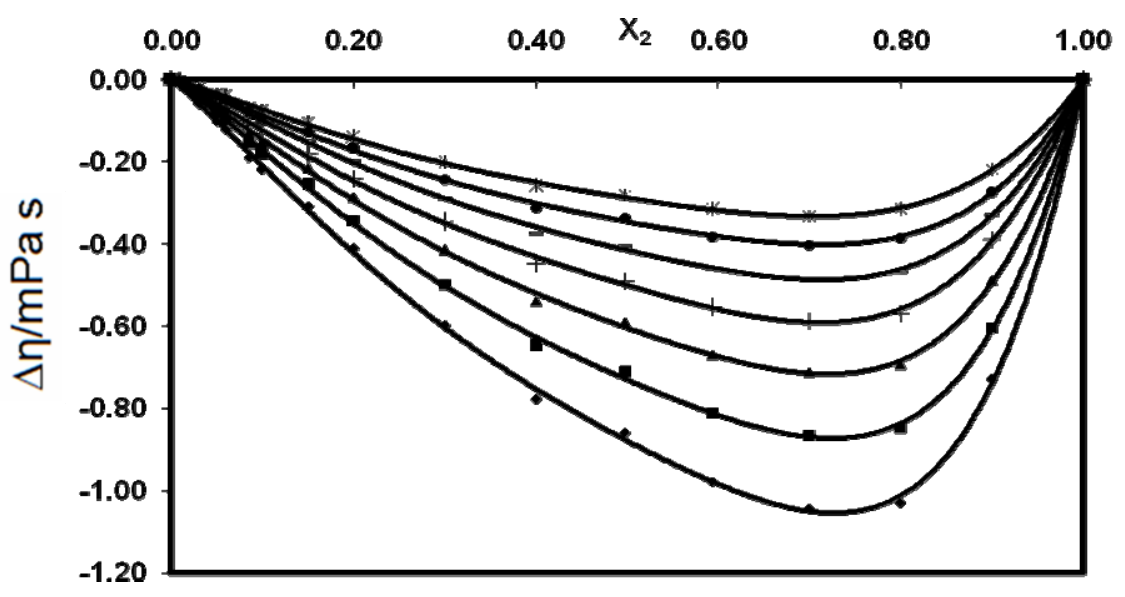

Fig.1: Desviaciones de viscosidad de la mezcla DMF +1-Butanol en función de la concentración a las temperaturas de $(\cdot 283.15, \mathbf{- 2 8 8 . 1 5}, \mathbf{\Delta} 293.15,+298.15,-303.15, \bullet 308.15, * 313.15) \mathrm{K}$

Las desviaciones de viscosidad obtenidas para esta mezcla fueron correlacionadas con la ecuación de Redlich-Kister, mediante la ecuación (2)

$\Delta \eta=x_{1} x_{2} \sum_{p=0}^{k} A_{p}\left(2 x_{1}-1\right)^{p}$

Donde $A_{p}$ son parámetros ajustables, $k$ es el número de parámetros. El ajuste fue realizado utilizando el método de los mínimos cuadrados, donde la desviación estándar $(\sigma)$ viene dada por la ecuación (3)

$\sigma=\left(\sum_{i=1}^{m} \frac{\left(\Delta \eta_{\text {exp }, i}-\Delta \eta_{c a l, i}\right)^{2}}{m}\right)^{1 / 2}$

En esta ecuación $\Delta \eta_{\text {exp,i, }} \Delta \eta_{c a l, i}$ son las desviaciones de viscosidad determinadas a partir de los datos obtenidos en este trabajo y calculados con la ecuación (2) respectivamente, $m$ es el número de puntos experimentales. Los resultados obtenidos se muestran en la tabla 2.

Tabla 2: Parámetros $A_{p}$ de la ecuación (2) y la correspondiente desviación estándar poblacional para el sistema binario DMF(1)+1-Butanol(2)

\begin{tabular}{cccccc}
\hline $\mathrm{T} / \mathrm{K}$ & $\mathrm{A}_{0}$ & $\mathrm{~A}_{1}$ & $\mathrm{~A}_{2}$ & $\mathrm{~A}_{3}$ & $\sigma / \mathrm{mPa} \mathrm{s}$ \\
\hline 283.15 & -3.51 & 2.33 & -2.70 & 2.05 & 0.01 \\
\hline 288.15 & -2.920 & 1.903 & -2.198 & 1.734 & 0.009 \\
\hline 293.15 & -2.417 & 1.545 & -1.750 & 1.358 & 0.008 \\
\hline 298.15 & -2.013 & 1.278 & -1.361 & 0.959 & 0.007 \\
\hline 303.15 & -1.668 & 1.020 & -1.140 & 0.909 & 0.006 \\
\hline 308.15 & -1.384 & 0.808 & -0.966 & 0.797 & 0.005 \\
\hline 313.15 & -1.153 & 0.674 & -0.726 & 0.553 & 0.004 \\
\hline
\end{tabular}


Siguiendo la aproximación de Eyring; la viscosidad absoluta $(\eta)$ de un fluido Newtoniano viene dada por la ecuación (4) (Páez et al., 2011)

$\ln \left(\frac{\eta V}{h N}\right)=\frac{\Delta H^{*}}{R T}-\frac{\Delta S^{*}}{R}$

Donde $h$ es la constante de Planck, $T$ es la temperatura absoluta, $N$ es el número de Avogadro, $V$ es el volumen molar del líquido y/o la disolución de trabajo, $\Delta H^{*}$ y $\Delta S^{*}$ son la entalpía y la entropía de activación para el proceso de flujo respectivamente.El gráfico del $\ln (\eta V / h N)$ contra $1 / T$ en cada punto de composición para el sistema binario en estudio muestra un comportamiento lineal en el intervalo de temperaturas estudiado, donde los valores de entalpia y entropía de activación fueron calculados a partir del correspondiente intercepto y la pendiente para cada linealización. Una vez obtenido estos valores se obtuvo la energía libre de activación $\Delta G^{*}$ mediante la ecuación (5).

$$
\Delta G^{*}=\Delta H^{*}-T \Delta S^{*}
$$

Los resultados que se obtienen bajo este tratamiento se muestran en la tabla 3.

Tabla 3: Parámetros de activación, $\Delta S^{*}, \Delta H^{*}, \mathrm{y} \Delta G^{*}$ para la mezcla $\mathrm{DMF}(1)+1$-Butanol(2) a varias temperaturas.

\begin{tabular}{|c|c|c|c|c|c|c|c|c|c|}
\hline & \multirow{2}{*}{\multicolumn{2}{|c|}{ 1-Butanol + DMF }} & \multicolumn{7}{|c|}{$\mathrm{T} / \mathrm{K}$} \\
\hline & & & 283.15 & 288.15 & 293.15 & 298.15 & 303.15 & 308.15 & 313.15 \\
\hline $\mathrm{X}_{2}$ & $\Delta H^{*} / \mathrm{kJmo}^{1} \pm 0.04$ & $\Delta S^{*} / \mathrm{Jmo}^{-1} K^{-1} \pm 0.14$ & \multicolumn{7}{|c|}{$\Delta G^{*} / \mathrm{kJ} \mathrm{mol}^{-1} \pm 0,0103$} \\
\hline 0.0000 & 8.52 & -13.41 & 12.317 & 12.383 & 12.449 & 12.519 & 12.582 & 12.652 & 12.720 \\
\hline 0.0100 & 8.67 & -13.25 & 12.371 & 12.436 & 12.501 & 12.567 & 12.632 & 12.697 & 12.763 \\
\hline 0.0305 & 8.73 & -12.96 & 12.402 & 12.466 & 12.531 & 12.596 & 12.661 & 12.726 & 12.790 \\
\hline 0.0501 & 8.86 & -12.66 & 12.444 & 12.507 & 12.570 & 12.634 & 12.697 & 12.760 & 12.823 \\
\hline 0.0601 & 8.92 & -12.54 & 12.467 & 12.530 & 12.592 & 12.655 & 12.718 & 12.781 & 12.843 \\
\hline 0.0856 & 9.06 & -12.11 & 12.491 & 12.552 & 12.613 & 12.673 & 12.734 & 12.794 & 12.855 \\
\hline 0.1000 & 9.15 & -11.91 & 12.523 & 12.582 & 12.642 & 12.701 & 12.761 & 12.821 & 12.880 \\
\hline 0.1498 & 9.54 & -11.03 & 12.662 & 12.717 & 12.772 & 12.827 & 12.883 & 12.938 & 12.993 \\
\hline 0.2002 & 9.77 & -10.58 & 12.769 & 12.822 & 12.875 & 12.928 & 12.981 & 13.034 & 13.086 \\
\hline 0.2999 & 10.39 & -9.24 & 13.008 & 13.054 & 13.100 & 13.146 & 13.192 & 13.239 & 13.285 \\
\hline 0.4000 & 10.97 & -8.03 & 13.248 & 13.288 & 13.328 & 13.368 & 13.408 & 13.449 & 13.489 \\
\hline 0.4972 & 11.86 & -5.98 & 13.557 & 13.587 & 13.616 & 13.646 & 13.676 & 13.706 & 13.736 \\
\hline 0.5931 & 12.67 & -4.27 & 13.876 & 13.898 & 13.919 & 13.940 & 13.962 & 13.983 & 14.004 \\
\hline 0.6988 & 13.59 & -2.25 & 14.228 & 14.240 & 14.251 & 14.262 & 14.273 & 14.285 & 14.296 \\
\hline 0.7990 & 14.52 & -0.31 & 14.610 & 14.611 & 14.613 & 14.614 & 14.616 & 14.617 & 14.619 \\
\hline 0.8990 & 16.42 & 4.33 & 15.197 & 15.175 & 15.154 & 15.132 & 15.110 & 15.089 & 15.067 \\
\hline 1.0000 & 18.88 & 10.29 & 15.961 & 15.910 & 15.859 & 15.807 & 15.756 & 15.704 & 15.653 \\
\hline
\end{tabular}


En la tabla 3 , se observa que los valores de $\Delta H^{*}$ para los componentes puros son positivos y varían en el siguiente orden: 1-Butanol > DMF lo que significa que la energía que requiere el 1Butanol para distorsionar sus enlaces es más grande en comparación con la DMF, indicando que la autoasociación del alcohol es mayor que para la DMF. Los valores de $\Delta S^{*}$ para los componentes puros, muestran que este es negativo para la DMF y positivo para el 1-Butanol, lo que indica que la formación del complejo activado se incrementa a medida que disminuye la concentración del 1-Butanol y disminuye con el aumento de la concentración del alcohol debido a la disrupción de los enlaces de hidrógeno. Los valores de $\Delta H^{*}$ y $\Delta S^{*}$ para la mezcla DMF (1) +1-Butanol(2) son positivos para todas las composiciones de estudio; el comportamiento positivo de los $\Delta G^{*}$ se debe a que la contribución entálpica en el sistema es mayor que la contribución entrópica y por lo tanto $\Delta G^{*}$ es positivo como se puede apreciar en la ecuación (5) lo cual podría indicar que las interacciones $\operatorname{DMF}(1)+1$-Butanol(2) se favorecen. Este tipo de comportamiento ha sido también observado en otros sistemas (Dumitrescu et al.,2004).

Sin embargo, el mejor criterio para determinar la naturaleza de la interacción entre los componentes de sistemas binarios es el signo de los parámetros de activación de exceso. Las funciones termodinámicas de exceso $\Delta G^{*}, \Delta H^{*} E, \Delta S^{*}$ fueron calculados mediante la ecuación(6)

$$
Y^{E}=Y-\left(x_{1} Y_{1}+x_{2} Y_{2}\right)
$$

Donde $Y$ es la medida de la propiedad termodinámica, $Y_{1}, Y_{2}, x_{1}$ y $x_{2}$ son las propiedades termodinámicas y las fracciones molares de los componentes (DMF y 1-Butanol) en la mezcla respectivamente.

Los resultados obtenidos para $\Delta H^{* E}$ y $\Delta S^{*}$ se muestran en la tabla 4.

Tabla 4: Entalpia de exceso $\Delta H^{*}$ y entropía de exceso $\Delta S^{*}$, para la mezcla $D M F(1)+1-B u t a n o l(2)$

\begin{tabular}{ccc}
\hline $\mathrm{X}_{2}$ & $\begin{array}{c}\Delta H^{*} \mathrm{E} / \mathrm{KJmol}^{-1} \\
\pm 0.04\end{array}$ & $\begin{array}{c}\Delta S^{*} \mathrm{E} / \mathrm{Jmol}^{-1} \mathrm{~K}^{-1} \\
\pm 0.14\end{array}$ \\
\hline 0.0100 & 0.05 & -0.08 \\
0.0305 & -0.10 & -0.27 \\
0.0501 & -0.18 & -0.44 \\
0.0601 & -0.23 & -0.55 \\
0.0856 & -0.34 & -0.73 \\
0.1000 & -0.40 & -0.87 \\
0.1498 & -0.53 & -1.17 \\
0.2002 & -0.82 & -1.91 \\
0.2999 & -1.23 & -2.94 \\
0.4000 & -1.69 & -4.10 \\
0.4972 & -1.81 & -4.36 \\
0.5931 & -1.99 & -4.91 \\
0.6988 & -2.17 & -5.41 \\
0.7990 & -2.27 & -5.84 \\
0.8990 & -1.41 & -3.57 \\
\hline
\end{tabular}

Los valores obtenidos para $\Delta G^{*} E$ se observan en la figura 2.

Si los valores para la energía libre de exceso $\Delta G^{*}$ son positivos se prevé interacción fuerte o muy fuerte entre componentes disimilares, dependiendo de su magnitud. Para valores negativos de la energía libre de exceso, por el contrario, indican interacción débil. Valores positivos $\Delta H^{*} E$ se asocian a interacciones fuertes especificas entre componente disimilares, mientras que valores negativos, por el contrario se asocian a interacciones débiles y valores positivos $\Delta S^{*} E$ indican una estructura más desordenada de las especies en el estado activado, mientras que los valores negativos indican que el estado activado es estructuralmente más ordenado que el estado fundamental (Tsierkezos et al.,2006). 
Como se observa en la tabla 4 y la figura 2 , la tendencia negativa de los de $\Delta H^{*} E$ y $\Delta G^{*}$ se atribuyen a interacciones débiles entre las moléculas de la DMF y las de 1-butanol, lo cual está en correspondencia con los resultados y análisis para la viscosidad de exceso. Por otro lado, el signo negativo del $\Delta S^{*}$ podría indicar que las especies formadas en el estado activado son estructuralmente más ordenadas que en el estado fundamental.

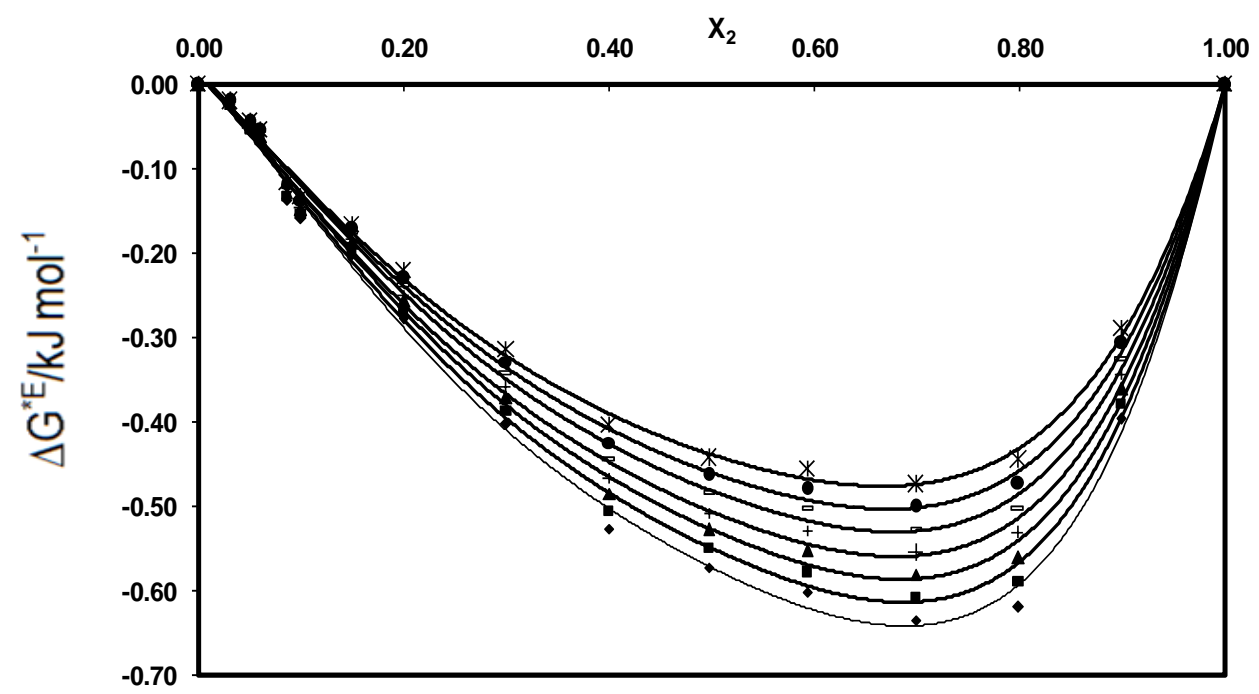

Fig.2: Energía libre de Gibbs de exceso en función de la fracción molar de 1-Butanol a las temperaturas de estudio $(\bullet 283.15,-288.15, \boldsymbol{\star} 293.15,+298.15,-303.15, \bullet 308.15, * 313.15) \mathrm{K}$

\section{CONCLUSIONES}

En este trabajo se reportan datos experimentales para la viscosidad absoluta y las desviaciones de viscosidad del sistema binario $\mathrm{DMF}(1)+1$-Butanol(2) en todo el intervalo de composición y temperaturas.

Las desviaciones de viscosidad de la mezcla binaria de (DMF)(1)+1-butanol(2) son negativas a todas las temperaturas de estudio y en todo el intervalo de concentración. Este comportamiento se asocia a la disrupción de los enlaces de hidrógeno en el alcohol y débiles interacciones físicas entre las especies de la mezcla, lo cual está en buen acuerdo con los valores obtenidos para los parámetros de activación del flujo viscoso. De forma análoga la tendencia negativa de los de $\Delta H^{*} E$ y $\Delta G^{*} E$ se atribuyen a interacciones débiles entre moléculas disimilares.

Finalmente el signo negativo del $\Delta S^{*}$ podría asociarse probablemente a que las especies formadas en el estado activado son estructuralmente más ordenadas que en el estado fundamental.

\section{AGRADECIMIENTOS}

Los autores agradecen a la universidad de Córdoba por el apoyo prestado para la realización de este trabajo

\section{REFERENCIAS}

Ali,A.,A.K. Nain,Physico-chemical studies of non-aqueous binary liquid mixtures at various temperatures. Thermochimica Acta: 274 209-221(1996). 
Bhuiyan, M.,M.Uddin,Excess molar volumes and excess viscosities for mixtures of N,Ndimethylformamide with methanol, ethanol and 2-propanol at different temperatures. Journal of Molecular Liquids: 138 139-146(2008).

Dumitrescu,V.,O. Pantea,Viscosities of binary mixtures of toluene with butan-1-ol and 2methylpropan-2-ol. J. Serb. Chem. Soc.: 70 (11) 1313-1323 (2005).

Kumar, A., Molecular interactions in binary mixtures of formamide with 1-butanol,2-butanol, 1,3butanediol and 1,4-butanediol at different temperatures: An ultrasonic and viscometric study. Fluid Phase Equilibria: 265 46-56(2008).

Mohammad, A., M Hemayet,viscosity and thermodynamics of viscous flow of the systems,1Propanol + formamide, $+\mathrm{N}$-methylformamide, $+\mathrm{N}, \mathrm{N}$-dimethylformamide. Physics and Chemistry of Liquids: 37 701-718(1999).

Páez, M., A. Portacio, C. Ortega,Propiedades Volumétricas y Viscosimétricas de Soluciones Acuosas Diluidas de 1,4 Pentanodiol a varias Temperaturas. Información Tecnológica: 22(1) 59-64 (2011).

Páez, M., J. Lafont, A. Alvis, Densidades y Propiedades Volumétricas de Mezclas N,NDimetilformamida + Agua a Presión Constante de $98.93 \mathrm{kPa}$ y a varias Temperaturas. Información Tecnológica: 20 (5) 47-54 (2009).

Páez, M., J. Lafont, A. Portacio, Efecto de la Temperatura sobre la Viscosidad deSoluciones Acuosas Diluidas de 1,2-Pentanodiol. Información Tecnológica: 20(2) 55-60 (2009).

Perry,R., Manual del Ingeniero Químico, 6a edición, volumen 3, 153-457,MacGraw-Hill, México(1992).

Riddick, J. A., Bunger, W. B., Sakano, T. K., Organic solvents: Physical properties and methods of purification, Techniques of Chemistry, $3^{a}$ edición,145-147.Wiley - Interscience, New York (1986).

Yang,C., Y. Sun, Y. He, y P. Ma.,Volumetric Properties and Viscosities of Binary Mixtures of N,NDimethylformamide with Methanol and Ethanol in the Temperature Range (293.15 to 333.15) K. J. Chem. Eng. Data: 53 293-297(2008).

Zivkovi'c ,E. y otros cuatro autores,Viscosity of the binary systems 2-methyl-2-propanol with nalkanes at $\mathrm{T}=(303.15,308.15,313.15,318.15$ and 323.15) $\mathrm{K}$ : Prediction and correlation - New UNIFAC-VISCO interaction parameters. Fluid Phase Equilibria: 299 191-197 (2010). 